The AstronomicAL JouRnal, 121:2804-2814, 2001 May

(C) 2001. The American Astronomical Society. All rights reserved. Printed in U.S.A.

\title{
THE INCLINATION DISTRIBUTION OF THE KUIPER BELT
}

\author{
MiChAEL E. BROWN \\ Division of Geological and Planetary Sciences, California Institute of Technology, Pasadena, CA 91125; mbrown@gps.caltech.edu \\ Received 2000 November 3; accepted 2001 February 1
}

\begin{abstract}
We develop a general method for determining the unbiased inclination distribution of the Kuiper belt using only the inclination and latitude of discovery of known Kuiper belt objects (KBOs). These two parameters are well determined for each discovered object, so we can use all 379 known KBOs (as of 2001 January 1) - without knowing the object's precise orbit, area, detection efficiency, or the latitudinal coverage of the survey that found the object - to determine the inclination distribution. We find that a natural analytic form for the inclination distribution is a sine of the inclination multiplied by a Gaussian. The inclination distribution of all KBOs is well fitted by $\sin i$ multiplied by a sum of two Gaussians with widths $2.6_{-.2}^{+.8}$ and $15^{\circ} \pm 1^{\circ}$. For this inclination distribution, the Kuiper belt has an effective area of $8100_{-1100}^{+1500} \mathrm{deg}^{2}$ and a FWHM of $12.5^{\circ} \pm 3.5^{\circ}$ in latitude. The inclination distribution of the different dynamical classes appear different. The Plutinos are well fit by $\sin i$ mulitplied by a single Gaussian of width $10.2_{-1.8}^{+2.5}$, the classical KBOs cannot be fit to a single Gaussian but are well fit by $\sin i$ multiplied by the sum of two Gaussians of widths $2.2_{-.6}^{+.2}$ and $17^{\circ} \pm 3^{\circ}$, and the scattered KBOs are poorly fit by $\sin i$ multiplied by a single Gaussian of width $20^{\circ} \pm 4^{\circ}$. The poor fit of the scattered objects is possibly a result of limitations of the method in dealing with large eccentricities. The effective areas of the Plutinos, classical KBOs, and scattered KBOs are $9300 \pm 1800,6100 \pm 2100$, and $17000 \pm 3000 \mathrm{deg}^{2}$, respectively. The FWHMs are $23^{\circ} \pm 5^{\circ}, 6.8_{-3.6}^{+2.0}$, and $44^{\circ} \pm 10^{\circ}$, respectively. In all cases, the inclinations of the Kuiper belt objects appear larger than expected from dynamical simulations of possible perturbations.
\end{abstract}

Key words: Kuiper belt - solar system: formation

\section{INTRODUCTION}

The discovery of Kuiper belt objects (KBOs) with inclinations as high as $30^{\circ}$ demonstrates that the full latitudinal extent of the Kuiper belt must be quite large. Understanding the true extent of the inclination distribution of KBOs is crucial to determining the total number of objects and the amount of mass sequestered in the belt, and also for understanding the dynamical processes operating in the current and past Kuiper belt. The distribution of observed KBO inclinations is a highly unrepresentative measure of the total Kuiper belt inclination distribution. Observations near the ecliptic are strongly biased toward discovery of low-inclination objects that spend the majority of their time close to the ecliptic, while observations off the ecliptic have zero probability of detecting objects with inclinations lower than the observed ecliptic latitude. The most straightforward method for determining the inclination distribution of the Kuiper belt would be to survey a range of ecliptic latitudes and measure the density of KBOs at each latitude. Unfortunately, such a dedicated survey with well-defined detection efficiency takes large amounts of telescope time and finds a limited number of objects, particularly at high latitudes, where the number densities are likely low.

As a more efficient alternative to such a direct method, we have developed a method for using every known KBOwithout knowing the object's precise orbit, area, detection efficiency, or the latitudinal coverage of the survey that discovered the object - to construct the inclination distribution of the Kuiper belt. The method relies on the fact that any KBO discovered can be considered an instantaneous probe of the inclination distribution at the latitude that the object is discovered. Thus, while the inclination of each $\mathrm{KBO}$ discovered is strongly biased by the ecliptic latitude of the observation, a disentanglement of the biases can allow us to reconstruct the true inclination distribution of the Kuiper belt. We are therefore able to use all detected KBOs to construct the distribution, greatly increasing the statistical meaningfulness of the distribution determined.

\section{DEFINITIONS AND DERIVATIONS}

The inclination distribution of the Kuiper belt is simply defined as the total distribution of inclinations of all objects in the belt. We use the phrase "total inclination distribution," or $f_{t}(i)$, to refer to this distribution. Another important distribution we discuss we refer to as the "ecliptic inclination distribution," or $f_{e}(i)$, which is the distribution of inclinations of KBOs instantaneously crossing the ecliptic at any time. As most KBO surveys target the ecliptic, the distribution of inclinations of the known KBOs (Fig. 1a) is similar to the ecliptic inclination distribution. An even better approximation to the ecliptic inclination distribution is the inclination distribution of only the KBOs that were discovered at a low-ecliptic latitude (Fig. 1b). Another related quantity is the distribution of the number of $\mathrm{KBOs}$ as a function of ecliptic latitude, which we will call the latitudinal distribution, $L(\beta)$ (note that this distribution differs from the distribution of the surface density by a factor of $\cos \beta$ ).

The relationships between these three distributions are easily derived for the case of purely circular orbits. The complication of elliptical orbits does not qualitatively alter the results and will be discussed later. For an object on a circular orbit with inclination $i$, the fraction of its orbit that the object is found at an ecliptic latitude less than or equal to $\beta$ is

$$
F(\beta)=\frac{2}{\pi} \sin ^{-1}\left[\min \left(\frac{\sin \beta}{\sin i}, 1\right)\right]
$$




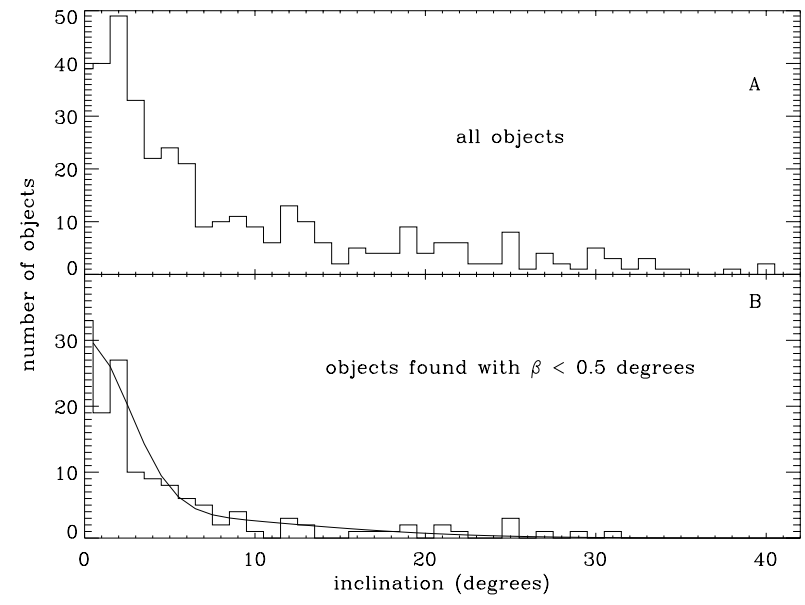

Fig. 1.-Inclination distribution of the known Kuiper belt. (a) The distribution of all 379 KBOs cataloged as of 2001 January 1 . This distribution is highly biased by the ecliptic latitude of the observations. (b) The distribution of $143 \mathrm{KBOs}$ found at ecliptic latitudes lower than 0.5 . The biases in this sample are quantifiable and can be removed to determine the total inclination distribution of the Kuiper belt. A fit to a sum of two Gaussians is shown as the solid line.

(Note that we are implicitly assuming that the geocentric latitude is equal to the heliocentric latitude. For distant objects like KBOs, this approximation holds closely.)

The latitudinal distribution for a single object with a single given inclination, $L_{i}$, is then simply the derivative of this cumulative distribution function:

$$
\begin{aligned}
L_{i}(\beta) & =\frac{d}{d \beta} F(\beta) \\
& =\frac{\cos \beta}{\left(\sin ^{2} i-\sin ^{2} \beta\right)^{1 / 2}} .
\end{aligned}
$$

At the ecliptic, $L_{i}=1 / \sin i$. If a collection of objects is found at the ecliptic with an ecliptic inclination distribution of $f_{e}(i)$, the total inclination distribution can now be calculated by weighting each detection by the inverse of the probability of finding that object at the ecliptic. Thus, we find that

$$
f_{t}(i)=\sin i f_{e}(i) .
$$

Note that any physical total inclination distribution must approach zero at an inclination of zero.

The latitudinal distribution is found by integrating equation (3) over the total inclination distribution:

$$
L(\beta)=\int_{\beta}^{\pi / 2} f_{t}(i) \frac{\cos \beta}{\left(\sin ^{2} i-\sin ^{2} \beta\right)^{1 / 2}} d i .
$$

To demonstrate the differences between the total inclination distribution, the ecliptic inclination distribution, and the latitudinal distribution, we will consider the hypothetical case of an isotropic distribution of objects. An isotropic distribution can be constructed by placing objects at an arbitrary location in space and allowing them to move in equally arbitrary directions. For such a distribution, the ecliptic inclination distribution is simple to determine: at the ecliptic, like at all other points, the direction of motion is arbitrary, thus the ecliptic inclination distribution is uniform. An isotropic distribution has a latitudinal distribution proportional to the surface area at each latitude; equa- tion (5) gives $L(\beta)=\cos \beta$. Finally, we find that $f_{t}(i)=\sin i$, as expected from the area of parameter space available to each inclination.

\section{METHOD}

\subsection{Data}

The key to the determination of the Kuiper belt inclination distribution is to collect the inclination of each known $\mathrm{KBO}$ and the heliocentric ecliptic latitude at the time of its discovery. These quantities are two of the most robust parameters known for a $\mathrm{KBO}$, even if the orbit is never precisely determined. Of objects recovered at a second opposition, the revised inclination has differed from the initial inclination estimate by more than $3^{\circ}$ only $4 \%$ of the time (Fig. 2).

The Minor Planet Center ${ }^{1}$ maintains a list of orbital elements and discovery dates of all known KBOs. The ecliptic latitude at the time of discovery is determined by taking these orbital elements and calculating the position of the object at the time of discovery. The ecliptic latitude at time of discovery versus inclination of the 344 objects cataloged as of 2000 October 1 is shown in Figure 3. The distribution of latitude versus inclination immediately shows the second bias mentioned above: at a particular latitude, $\beta$, no object will ever be discovered with an inclination, $i$, such that $i<\beta$. In addition to this obvious bias, one can also see from the distribution that observations at a ecliptic latitude $\beta$ are biased toward finding objects with inclinations close to that latitude, as is apparent from equation (1).

\subsection{Approximation of the Ecliptic Inclination Distribution}

The ecliptic inclination distribution, $f_{e}(i)$, is equal to the inclination distribution of all objects that are crossing the ecliptic at any moment. We can simply approximate $f_{e}(i)$ by determining the inclination distribution of all objects that

${ }^{1}$ See cfa-www.harvard.edu/iau/Ephemerides/Distant/index.html.

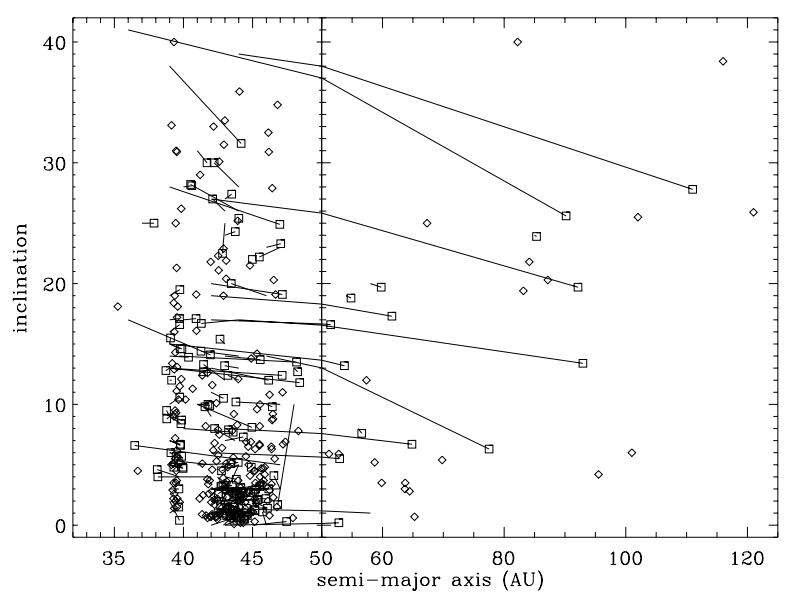

FIG. 2.-Inclination vs. semimajor axis for the known Kuiper belt. KBOs observed on only a single opposition are shown as diamonds. KBOs observed over multiple oppositions are show as squares. The lines show the inclination and semimajor axis initially reported for each multiopposition object. Most multiple opposition KBOs have inclinations within a few degrees of the initially reported value. The different dynamical classes appear to have different inclination distributions in this highly biased plot. $2000 \mathrm{CR} 105$, with a semimajor axis of $675 \mathrm{AU}$ and an inclination of 22.8 , falls off the plot. 


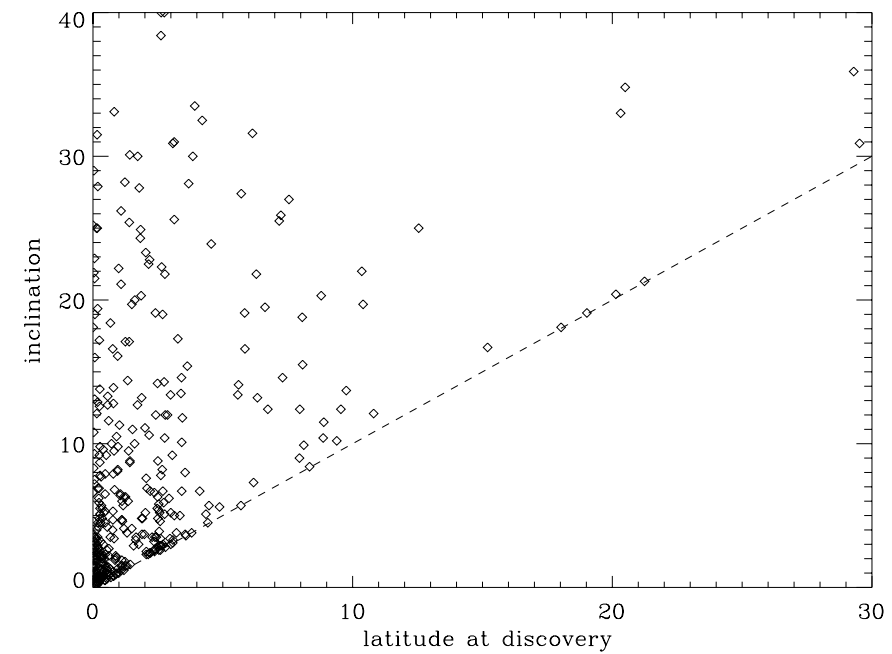

FIG. 3.-Ecliptic latitude at time of discovery vs. inclination for 379 KBOs. Two biases are obvious: no object can be detected with an inclination lower than the latitude, and observations at all latitudes find more objects with inclinations close to the latitude of observation.

were detected at the ecliptic. In practice, we will use all objects found within 0.5 of the ecliptic. Figure $1 b$ shows this approximation of $f_{e}(i)$ for the 143 known KBOs discovered near the ecliptic. This approximation of $f_{e}(i)$ is well fitted by a sum of two Gaussians,

$$
f_{e}(i)=a \exp \left(\frac{-i^{2}}{2 \sigma_{1}^{2}}\right)+(1-a) \exp \left(\frac{-i^{2}}{2 \sigma_{2}^{2}}\right),
$$

where $a=0.89, \sigma_{1}=2.7$, and $\sigma_{2}=13.2$ (see Fig. $1 b$; note that we do not normalize any of our distributions. To do so, divide each by its integral from $0^{\circ}$ to $180^{\circ}$ ). The total inclination distribution, $f_{t}(i)$, is simply found by multiplying this equation by $\sin i$,

$$
f_{t}(i)=\sin i\left[a \exp \left(\frac{-i^{2}}{2 \sigma_{1}^{2}}\right)+(1-a) \exp \left(\frac{-i^{2}}{2 \sigma_{2}^{2}}\right)\right],
$$

thus we have an initial estimate of the total inclination distribution.

A Gaussian appears to be a natural functional form for $f_{e}(i)$. We have performed Monte Carlo simulations of multiple perturbations in an initially zero inclination disk and find that $f_{e}(i)$ is perfectly fitted by such a distribution, with the width related to the number and magnitude of the individual perturbations. In addition, the distribution of inclinations of the known asteroids also appears to be moderately well fitted by a single Gaussian functional form. Such a distribution has several interesting functional properties: the distribution of vertical ecliptic crossing velocities, $H\left(v_{z}\right)$, is equal to $f_{t}(i)$ multiplied by $\sin i$, or

$$
H\left(v_{z}\right)=\sin ^{2} i \exp \left(\frac{-i^{2}}{2 \sigma^{2}}\right),
$$

which for the small values of $i$ typical of most KBOs is functionally equivalent to a Maxwellian velocity distribution. For large values of $\sigma$, the distribution approaches an isotropic distribution. For all these reasons, we deem $\sin i$ multiplied by a Gaussian to be the simplest natural functional form for the total inclination distribution. Note again that any total inclination distribution must approach zero at zero inclination. That the Kuiper belt inclination dis- tribution is fit by a sum of two Gaussians, rather than by a single Gaussian, suggests the existence of at least two classes of objects with distinct dynamical histories.

The approximation of $f_{t}(i)$ determined above, while useful for getting a general feeling for the shape, is not ideal. Only 143 of the 379 known objects are used, so statistics are not as good as if all objects could be used. In particular, the number of objects at high inclination is small because of the biases. If we were to divide the known objects into different dynamical classes, the numbers would be even smaller and the results would be correspondingly poorer. In addition, small problems are caused by the fact that the objects used for the distribution are not precisely at the ecliptic. The number of objects with inclinations less than about 0.5 should actually be higher, but we are biased against them. For these reasons, we have developed a more general method of determining the inclination distribution that allows us to use all the data available.

\subsection{Calculation of the Total Inclination Distribution}

No immediately obvious method exists to invert $f_{t}(i)$ directly using all the data. We resort instead to solving the forward problem; we take a series of hypothetical distributions and determine the probability that the observed objects could have been drawn from such distributions. From these probabilities, we can then determine the best fit of the hypothetical distributions, along with ranges of acceptable solutions.

To determine the probability that the observed objects could be drawn from a hypothetical inclination distribution, we first calculate, for each of $N$ known KBOs, the probability that object $j$, discovered at latitude $\beta_{j}$, would have an inclination of the observed inclination $i_{j}$ or lower, which is given by

$$
\begin{aligned}
P_{j}= & \int_{\beta_{j}}^{i_{j}} \frac{f_{t}\left(i^{\prime}\right)}{\left(\sin ^{2} i^{\prime}-\sin ^{2} \beta_{j}\right)^{1 / 2}} d i^{\prime} \\
& \times\left[\int_{\beta_{j}}^{\pi / 2} \frac{f_{t}\left(i^{\prime}\right)}{\left(\sin ^{2} i^{\prime}-\sin ^{2} \beta_{j}\right)^{1 / 2}} d i^{\prime}\right]^{-1} .
\end{aligned}
$$

If the hypothetical distribution is close to the true distribution, the collection of values of $P_{N}$ for all objects should be evenly distributed between 0 and 1 . (This point is immediately obvious by considering the problem in reverse; if one wanted to perform a Monte Carlo simulation of inclinations of KBOs chosen from a particular distribution, one would choose random numbers evenly distributed between 0 and 1 and assign an inclination equal to the inclination at which the probability of finding that inclination or lower is equal to the random number. We instead know the inclination and find the random number that would have had to be chosen to get that inclination.) We now need to apply a statistical test to determine if the numbers are evenly distributed. A familiar statistical test is the $\chi^{2}$ test. We could perform this test by binning the values of $P_{N}$ into, e.g., 0.1 wide bins and determining the probability that the number of values in those bins could have been chosen from a uniform sample. A good fit, in this case, would be one in which the $\chi^{2}$ test cannot rule out the hypothetical distribution with a high degree of confidence. A more powerful statistical test for this particular case is the KolmogorovSmirnov (K-S) goodness-of-fit test, which is designed to test continuous distributions (such as the values of $P_{N}$ ) without 
losing information by binning (Press et al. 1992). The K-S test is simple to implement. The statistic $D$ is calculated by comparing the sorted values of $P_{N}$ with the expected cumulative distribution function $S(x)$, which in our case is simply a set of evenly spaced numbers between 0 and 1 or $S(x)=$ $j / N$, so

$$
D=\max \left|P_{j}-j / N\right| .
$$

In practice, we use the Kuiper modification of the K-S test to correct the problem of the otherwise unequal weighting of $P_{N}$ (Press et al. 1992).

Just as in a $\chi^{2}$ test, the $D \sqrt{N}$ statistic, where $N$ is the number of objects in the sample, is calibrated to determine the probability that the hypothesis that the values of $P_{N}$ were drawn from a uniform sample can be rejected. We empirically calibrate $D \sqrt{N}$ by creating 100,000 realizations of random numbers uniformly distributed between 0 and 1 and calculating the probability of obtaining a particular value of $D \sqrt{N}$ when we know that the distribution is uniform (Fig. 4).

Unfortunately, while the integrals in equation (9) must be finite, we have been unable to find a numerical integration scheme that allows us to accurately integrate through the singularity at the outer limit. In practice, we therefore calculate the values of $P_{N}$ through a Monte Carlo simulation. For a given hypothetical inclination distribution, we randomly choose $10^{5}$ inclinations from the distribution and distribute the objects randomly within circular orbits. For each real known $\mathrm{KBO}$ with inclination $i$, we then calculate $P_{N}$ by constructing an empirical inclination distribution of Monte Carlo objects within 0.5 in latitude of the discovery position of the known object and then determining the probability that an object at the discovered latitude will have an inclination of $i$ or lower.

As an example, we construct a distribution of KBOs using $\sin i$ multiplied by the sum of Gaussians for $f_{t}(i)$ that we found above and compare it with the distribution of known KBOs. The distribution of the values of $P_{N}$ are shown in Figure 5a, along with the expected distribution for a randomly distributed values (straight line). The K-S test

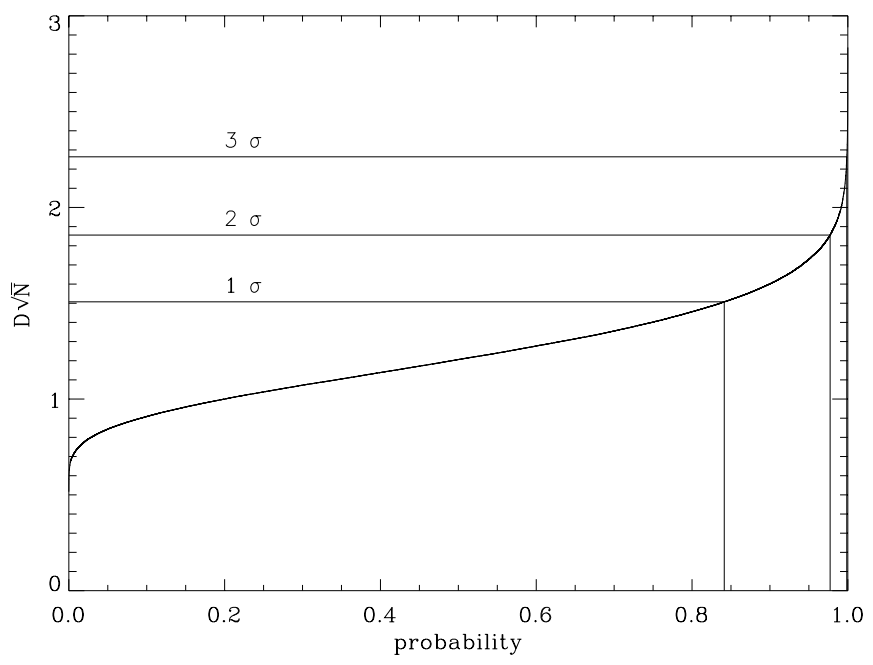

FIG. 4. - Calibration of the $D \sqrt{N}$ statistic for the K-S test. The plot shows the probability that the K-S test will yield a value of $D \sqrt{N}$ or higher when comparing two uniform distributions. The 1,2 , and $3 \sigma$ lines show the values at which the probability of exceeding $D \sqrt{N}$ is $15.9 \%, 2.3 \%$, and $0.1 \%$, respectively.

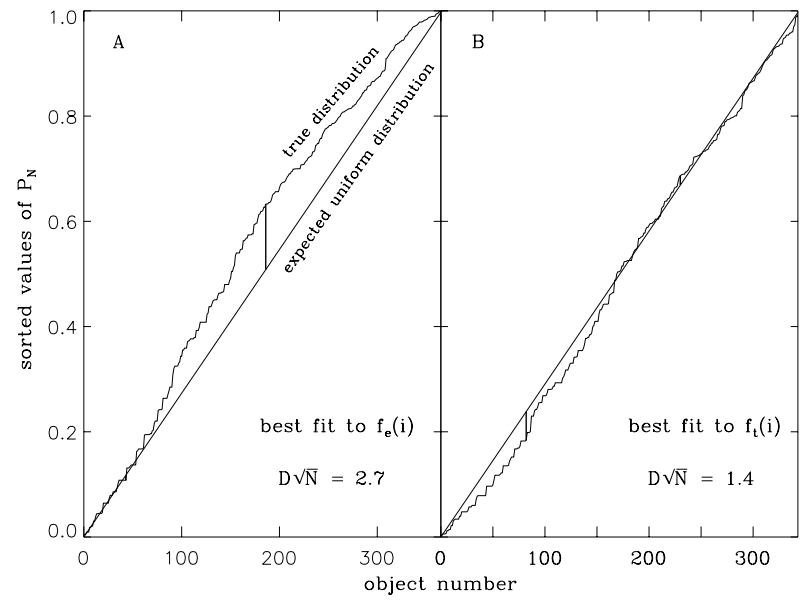

FIG. 5.- Calculation of the Kuiper variant of the K-S test. Here $D$ is simply the sum of the largest positive and negative excursions of the sorted values of $P_{N}$ away from the straight line. (a) Using the sum of Gaussians found for $f_{e}(i)$ gives a fit that can be rejected at the $99.998 \%$ confidence level, but a search of nearby parameter space yields a sum of Gaussian fits that can only be rejected at a $25 \%$ confidence level $(b)$ and is thus a statistically acceptable fit.

concludes that even though the $f_{e}(t)$ fit looks moderately good, the probability that the values of $P_{N}$ could have been chosen from a uniform distribution-and thus that the observed KBOs could have been chosen from this inclination distribution - is only $0.002 \%$. We can therefore reject this distribution at the $99.998 \%$ confidence level. A search of nearby parameter space finds best-fitted values of $a=0.83, \sigma_{1}=2.6$, and $\sigma_{2}=15^{\circ}$ that can only be rejected at the $25 \%$ confidence level and thus are a statistically good fit to the data. For such a distribution, almost 3 times as many objects are in the broad part of the distribution as are in the narrow part.

\section{ANALYSIS}

The known Kuiper belt objects naturally fall into three dynamical classes (Jewitt \& Luu 2000): the resonant KBOs locked into orbital resonance with Neptune, the classical $\mathrm{KBOs}$ in lower eccentricity orbits beyond $40 \mathrm{AU}$, and the scattered KBOs with high eccentricity and large semimajor axes. A plot of inclination versus semimajor axis of all the known objects suggests that the different KBO dynamical classes have different inclination distributions (Fig. 2). We therefore analyze these different classes separately.

The separation between the dynamical classes is best seen in a plot of semimajor axis versus eccentricity (Fig. 6). The most obvious class is the Plutinos with semimajor axes of about 39 AU in 3:2 resonance with Neptune. Other resonant objects include the objects clustered at $48 \mathrm{AU}$, which are in a 2:1 resonance with Neptune. Outside of $50 \mathrm{AU}$ appears another obvious class, the scattered KBOs, which mostly have high eccentricities and perihelia between 30 and $40 \mathrm{AU}$. In addition to objects with these perihelia, two eccentric objects appear with even closer perihelia, 2000 EE173 with a perihelion of $22 \mathrm{AU}$ and 1999 TD10 at 12 AU. These two objects appear to fit in more naturally with the nearer Centaurs, so we will exclude them as members of the scattered objects. The absence or presence of these objects does not affect any of the final results. Scattered objects need not have semimajor axes beyond 50 AU. Several objects between 40 and $50 \mathrm{AU}$ appear at distinctly 
higher eccentricity than the remainder of the population, and have perihelia in the same range as the scattered KBOs. We include these in our listing of the scattered objects. Again, this inclusion does not affect final results. The remaining objects distributed between 40 and $48 \mathrm{AU}$ in low eccentricity orbits are the classical KBOs.

\subsection{Resonant KBOs}

Of the resonant KBOs, only the Plutinos have a large enough known population to allow sufficient statistics to try to determine the inclination distribution. As a first approximation of the ecliptic inclination distribution, we look at the distribution of the 20 Plutinos found at latitudes less than 0.5 (Fig. 7). This distribution can be fitted by a single Gaussian with $\sigma=7.4$. We therefore try a singleGaussian fit multiplied by $\sin i$ for the total inclination distribution and perform our full Monte Carlo method. Figure 8 shows the value of the $D \sqrt{N}$ statistic of the K-S test, along with the confidence levels. The best-fit value, along

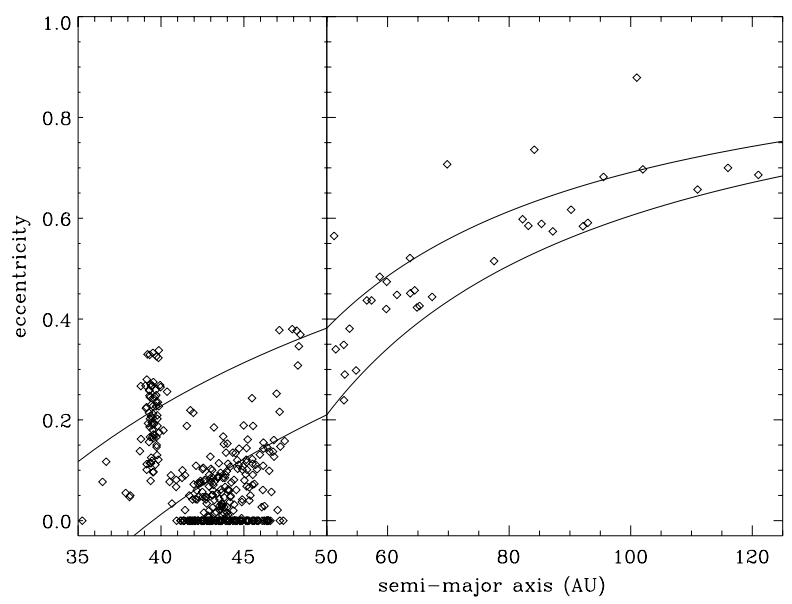

FIG. 6.-Eccentricity vs. semimajor axis for the known Kuiper belt. 2000 CR105, with a semimajor axis of 675 AU and an eccentricity of 0.94 , falls off the plot. The two solid lines show the parameter space for which the perihelion distance of the object ranges between 30.9 and $39.5 \mathrm{AU}$. The objects with zero eccentricity have poorly determined orbits that are assumed to be circular.

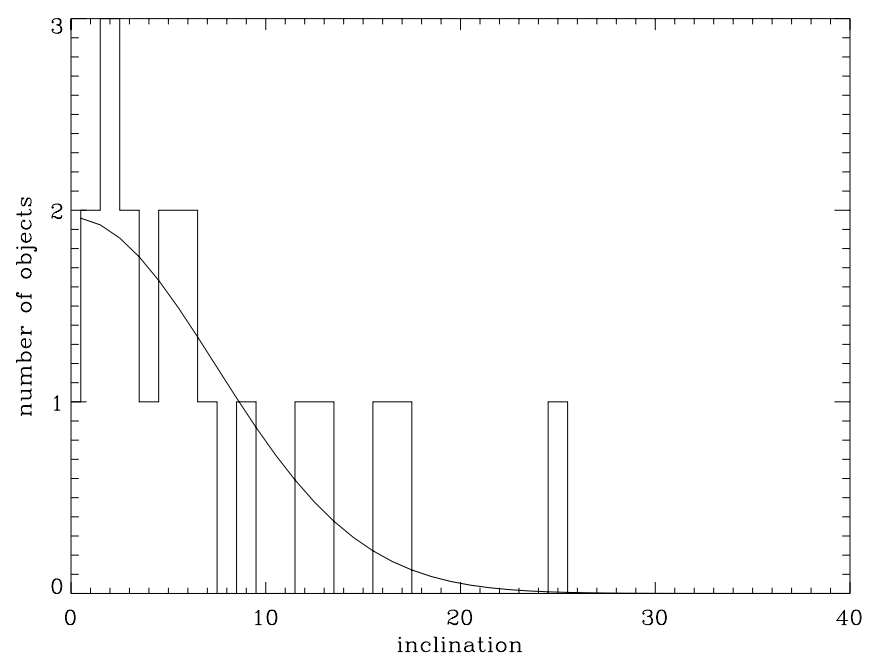

FIG. 7.- Inclination distribution of 20 Plutinos found at latitudes lower than 0.5 . The solid line shows the best single-Gaussian fit to the data, with $\sigma=7.4$. with $1 \sigma$ confidence bounds, gives a Gaussian width of $\sigma=$ $10^{\circ} 2_{-1.8}^{+2.5}$, in close agreement with our estimate from the observed ecliptic inclination distribution. The $1 \sigma$ error bars give the values with which we can reject the distribution at the $1 \sigma(84.1 \%)$ confidence level.

Given such an inclination distribution, we can now calculate the ecliptic inclination and latitudinal distributions for the best-fit model, along with the $1 \sigma$ extremes (Fig. 9). From the latitudinal distributions, we find that the total number of Plutinos is given by the density at the ecliptic multiplied by $8600 \pm 1800$ or, alternatively, the effective area of sky covered by the Plutinos is $9300 \pm 1800 \mathrm{deg}^{2}$, and the full width at half-maximum (FWHM) of the apparent distribution of Plutinos in the sky is $23^{\circ} \pm 5^{\circ}$.

\subsection{Classical KBOs}

We estimate the ecliptic inclination distribution of the classical KBOs using the 111 objects found within 0.5 of the ecliptic. A single Gaussian is a poor fit to the data (Fig. 10). A much better fit can be found from a sum of two Gaussians with $a=0.90, \sigma_{1}=2.2$, and $\sigma_{2}=8.8$. This distribution appears different from that of the resonant KBOs. The K-S test can also be used to calculate the probability that these two sets of inclinations were drawn from the same distribution. The value of the $D \sqrt{N}$ statistic from this test is 1.68 , which suggests that we can reject the hypothesis that the two distributions are drawn from the same intrinsic distribution at a $93.4 \%$ confidence level. While this probability is sufficiently high to rule out the hypothesis that the inclination distributions are the same at the $1 \sigma$ level, we cannot be any more confident than that. Nonetheless, we deem this rejection level sufficiently significant, and we will treat the classical objects separately.

As with the Plutinos, we first try to fit the inclination distribution of the classical KBOs with a single Gaussian multiplied by sine distribution. The full method shows that even the best-fit value of $\sigma=5.2$ can be ruled out at the greater than $99.999 \%$ confidence level. Guided by the twoGaussian fit to the ecliptic inclination distribution above, we try a similar two-Gaussian multiplied by $\sin i$ fit to the

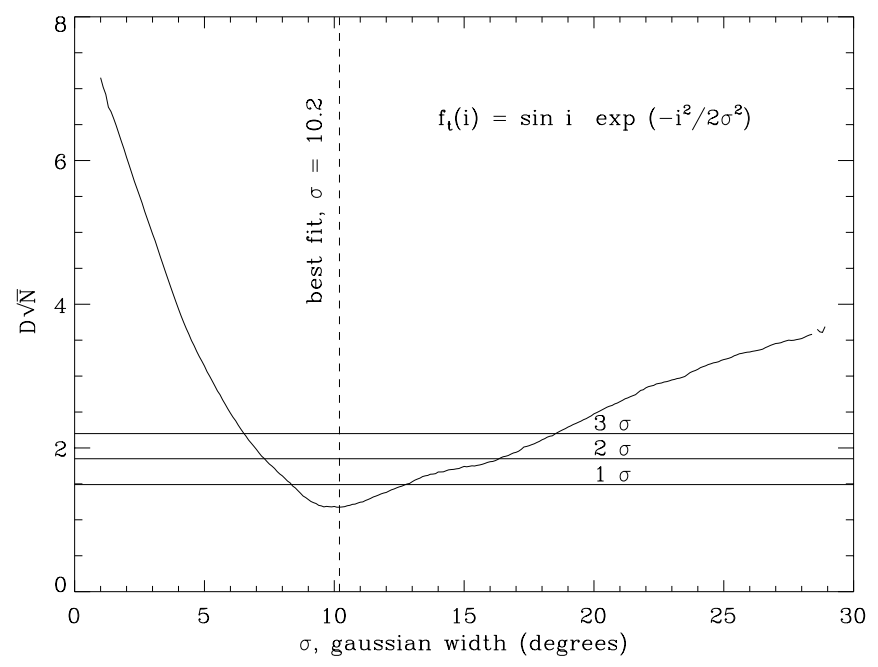

FIG. 8. $-D \sqrt{N}$ statistic as a function of $\sigma$ for distributions of the form $f_{t}(i)=\sin i \exp \left(-i^{2} / 2 \sigma^{2}\right)$ for Plutinos. The horizontal lines show the value of $D \sqrt{N}$ at which we can reject the hypothesis at the 1,2 , and $3 \sigma$ level. The best-fit value of $\sigma=10: 2$ can only be rejected with $43 \%$ confidence and is thus a statistically good fit to the data. 


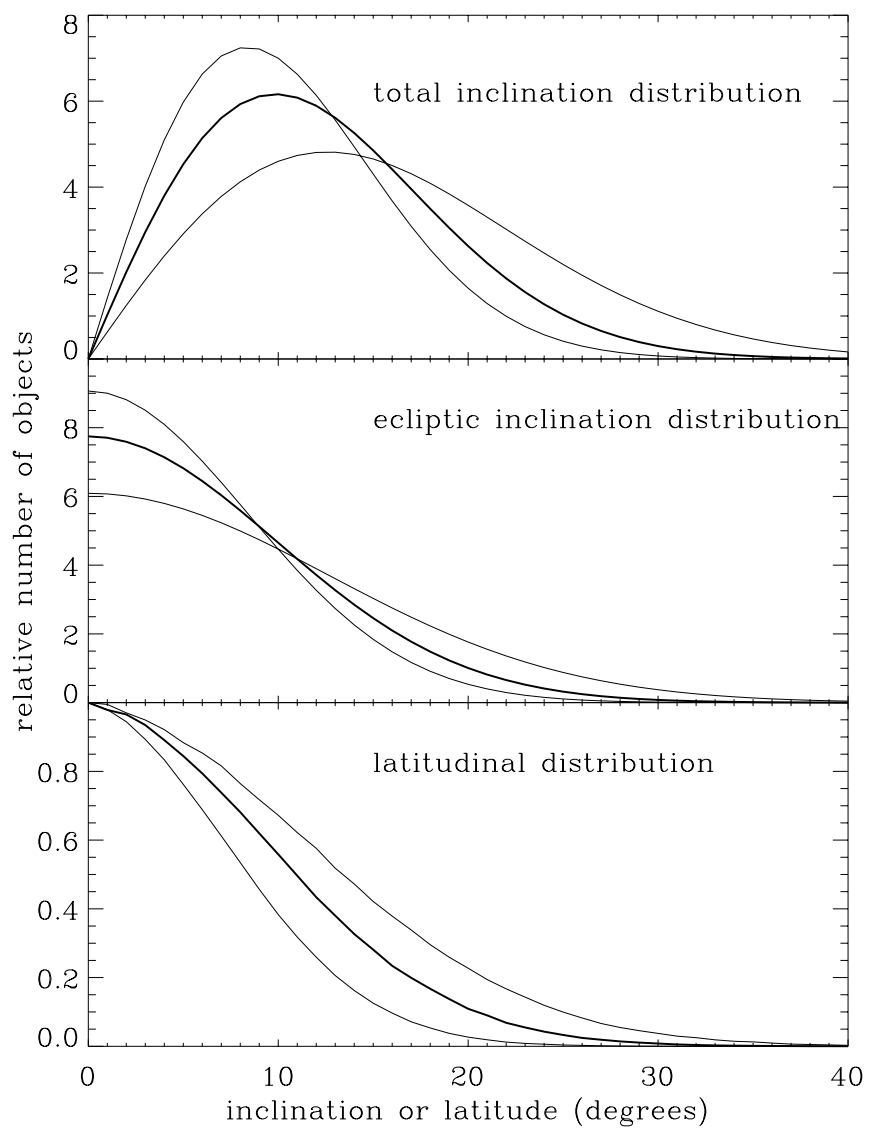

FIG. 9.- Total inclination, ecliptic inclination, and latitudinal distribution as a function of angle (inclination or, in the case of the latitudinal distribution, latitude). The thick lines show the best-fit distributions, while the thinner lines show the $1 \sigma$ variations. The inclination distributions are normalized, so that the total number of objects (the integral under the curve) is constant. The latitudinal distribution is normalized to a fixed value at the ecliptic.

total inclination distribution. The best fit for this case gives $a=0.93, \sigma_{1}=2.2$, and $\sigma_{2}=18^{\circ}$, with a $D \sqrt{N}$ statistic of 0.89 , implying a statistically good fit. For such a threeparameter fit, the values of $D \sqrt{N}$ form a three-dimensional volume, with all the values within the volume enclosed by the surface $D \sqrt{N}=1.49$ acceptable fits at the $1 \sigma$ level. If the three parameters were uncorrelated, we could simply give $1 \sigma$ error bars on each to indicate the range of acceptable values. Unfortunately, the parameters are highly correlated. We instead show the range of possible parameters in two ways. First, we give a series of contour plots, which are slices through the three-dimensional $D \sqrt{N}$ volume (Fig. 11). Each slice gives contours of confidence levels as a function of $\sigma_{1}$ and $\sigma_{2}$ for a given value of $a$. From these contour slices, the confidence of rejection of any particular sum of two Gaussians can be determined. In addition, to graphically demonstrate the range of acceptable values, we calculate the $1 \sigma$ variation in each parameter, keeping the other two parameters fixed. From this calculation, we find $a=0.93 \pm 0.02, \sigma_{1}=2.2_{-.6}^{+.2}$, and $\sigma_{2}=17^{\circ} \pm 3^{\circ}$. For the best fit, $19 \%$ of the objects are included in the narrow Gaussian distribution, while the remaining $81 \%$ are in the wider Gaussian. In Figure 12, we plot the best-fit inclination distribution, along with the effects of varying the parameters in these ways. Note, however, that these are not the full ranges allowed for these parameters, only the full range

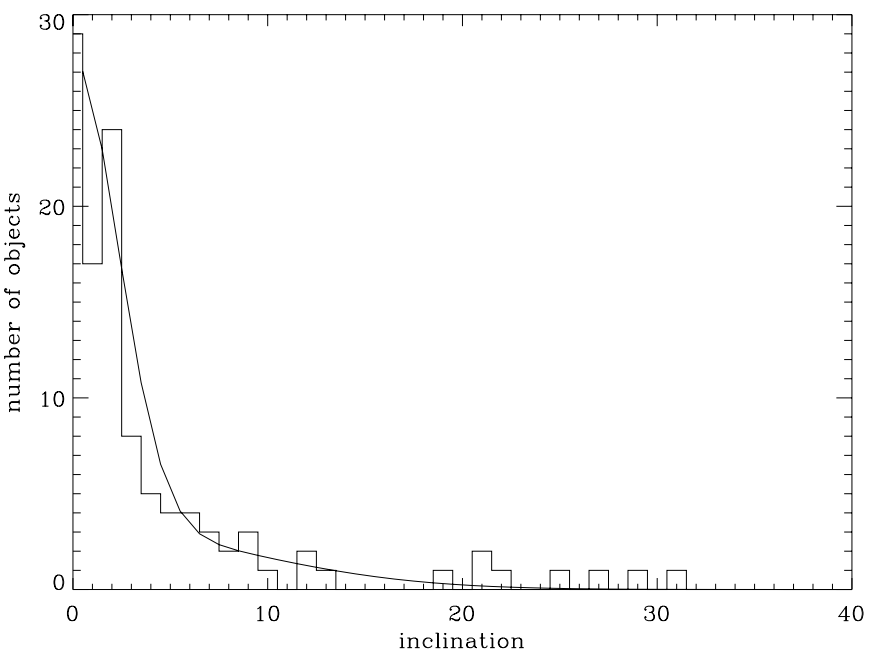

FIG. 10.-Inclination distribution of all 111 classical KBOs found at ecliptic latitudes lower than 0.5 . The solid line shows the best fit to the data using the sum of two Gaussians.

allowed with the other parameters fixed. Alternatively, if we calculated the full range allowed, we would find $a=$ $0.93 \pm 0.04, \sigma_{1}=2.2_{-1.2}^{+0.6}$, and $\sigma_{2}=18^{\circ} \pm 8^{\circ}$, but, again, these parameters cannot be varied independently by these amounts. While these two methods allow a more intuitive feel into the allowed ranges, the contour plots should be used to examine any particular set of parameters.

From the best-fit inclination distribution, we also calculate the ecliptic inclination distribution and the latitudinal distribution (Fig. 12). From the latitudinal distribution, we find an effective area of the classical Kuiper belt of $6100 \pm 2100 \mathrm{deg}^{2}$ and a FWHM of $6.8_{-3.6}^{+2.0}$ (allowing the full range of parameters). The extremely narrow FWHM is a consequence of the large number of objects in the narrower of the two Gaussian distributions.

The most noticeable characteristic of the distributions is the sharp break in slope in both the ecliptic inclination distribution and the latitudinal distribution, caused by the sum of two Gaussians. To determine whether this break in slope is actually present in the real distributions or an artifact caused by our choice of functional forms, we attempted a set of fits to the data using a distribution with no such hard break. We constructed distributions similar to the sum of two Gaussians, but we artificially connected the peaks of the two Gaussians in $f_{t}(i)$ with a straight line. These distributions lack the depletion in moderate inclination objects characteristic of the sums of Gaussians seen in Figure 12. We find that these distributions cannot be ruled out at the $1 \sigma$ level, thus we conclude that the change in slope evident in the distributions in Figure 12 is not required by the data. The real distributions need not be purely the sum of two Gaussians, but this analytic form is a simple approximation that captures the important behavior of the distribution. Nevertheless, we point out that the ecliptic inclination distribution (Fig. 10) appears well fit by a sum of two distinct Gaussians, with a sharp break between them.

\subsection{Scattered KBOs}

The small number of known scattered KBOs makes the determination of the inclination distribution least certain. Only 10 scattered KBOs have been found within 0.5 of the ecliptic (Fig. 13), so the estimate of the ecliptic inclination 


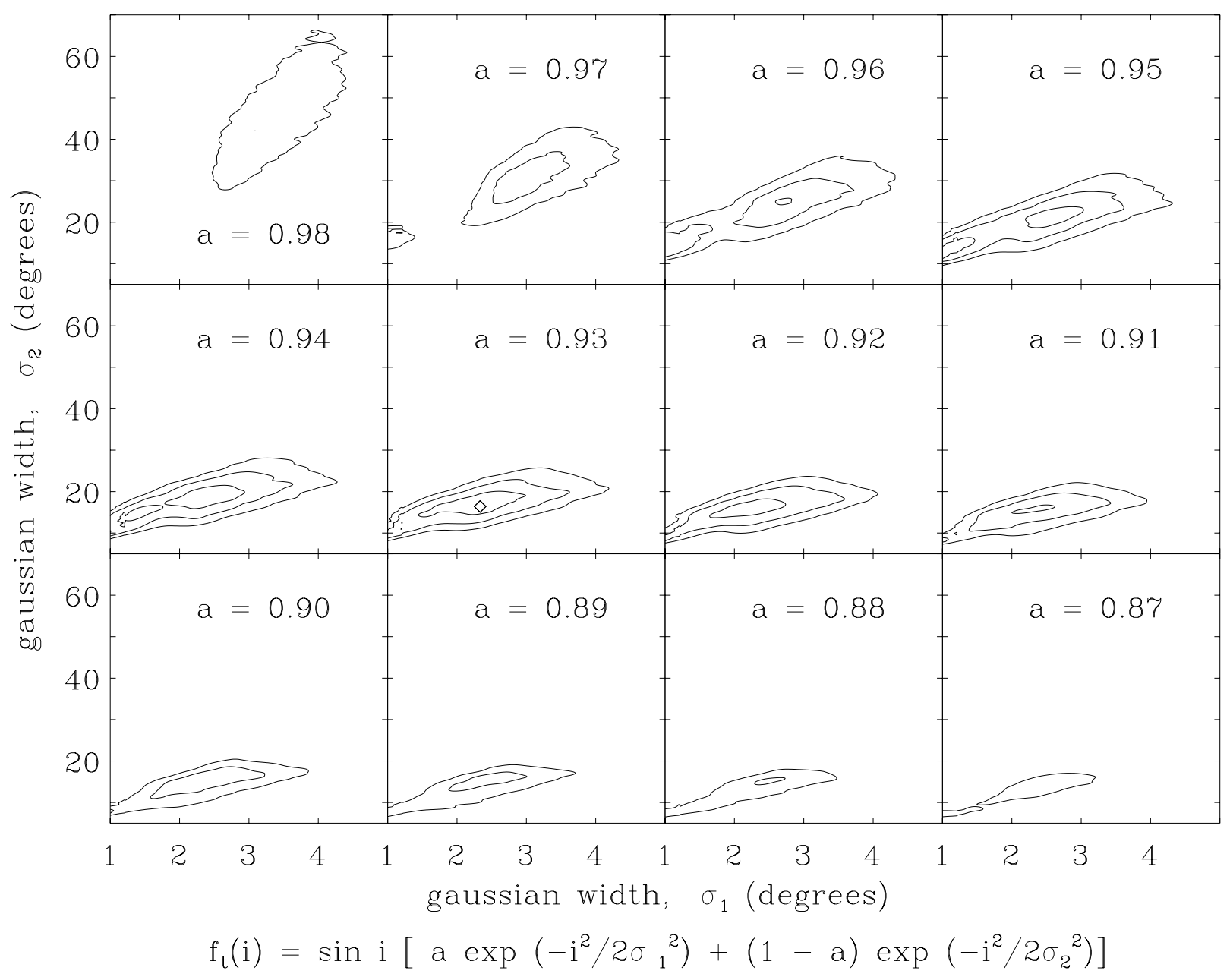

FIG. 11.-Confidence levels for a fit to the total inclination distribution of the classical Kuiper belt objects. The plots show the 1,2, and $3 \sigma$ contours of slices of the three-dimensional parameter space. The best-fit values of $a=0.93, \sigma_{1}=2.2$, and $\sigma_{2}=17^{\circ}$ are shown as a diamond.

distribution is particularly bad. Based on our previous findings and expectations, however, we attempt to fit the total inclination distribution with a single Gaussian multiplied by $\sin i$. The best fit gives a Gaussian width of $20^{\circ} \pm 4^{\circ}$, where now we have given $2 \sigma$ error bars, since none of the fits is acceptable at the $1 \sigma$ level. An attempt at a sum of two Gaussians multiplied by $\sin i$ for the total inclination distribution does not improve the fit significantly. The poor fit to the inclination distribution of the scattered KBOs could be due to the effects of the assumption of circular orbits, which is clearly incorrect for this class of objects. We discuss this possibility further below.

We show below that even though the fit for higheccentricity groups of objects is poor, the final result is reasonably accurate. We thus make the assumption that our fit of the total inclination distribution to a single Gaussian multiplied by $\sin i$ is representative of the true inclination distribution of the scattered KBOs. The fitted total inclination distribution, ecliptic inclination distribution, and latitudinal distribution, along with formal $2 \sigma$ error limits, are show in Figure 14. We find that the effective area of the scattered Kuiper belt is $17000 \pm 3000 \mathrm{deg}^{2}$, and the FWHM is $44^{\circ} \pm 10^{\circ}$, where we again present formal $2 \sigma$ error bars. Trujillo, Jewitt, \& Luu (2000) modeled the inclination distribution of the scattered Kuiper belt from the first four scattered objects recognized. While they used an unphysical total inclination distribution for their model (a Gaussian), they also comment that the data can be fitted by a total inclination distribution that is a Gaussian centered at $20^{\circ}$. Such a form would closely approximate our best-fit distribution.

\subsection{Limitations}

A large limitation to this method is the assumption that all orbits are strictly circular. For the Plutinos and the scattered KBOs, this assumption is far from correct. We explore errors caused by this assumption by constructing a variety of artificial plutino and scattered populations, simulating observations of these populations, and trying to determine the inclination distributions using our method.

For the Plutinos, we construct a Monte Carlo population of $10^{6}$ objects with semimajor axis chosen uniformly between 38.5 and 39.5 , eccentricity chosen uniformly between 0.07 and 0.35 (Fig. 6), and all other orbital parameters (except for inclination) randomly chosen between $0^{\circ}$ and $360^{\circ}$. We select a size for each object using a power-law size distribution, with a differential size index of 3.6 (Chiang $\&$ Brown 1999), and we calculate the brightness from the absolute magnitude and the geocentric distance (assuming all objects are observed approximately at opposition). To simulate the observations, we randomly select a Monte Carlo KBO having the same magnitude and latitude as 


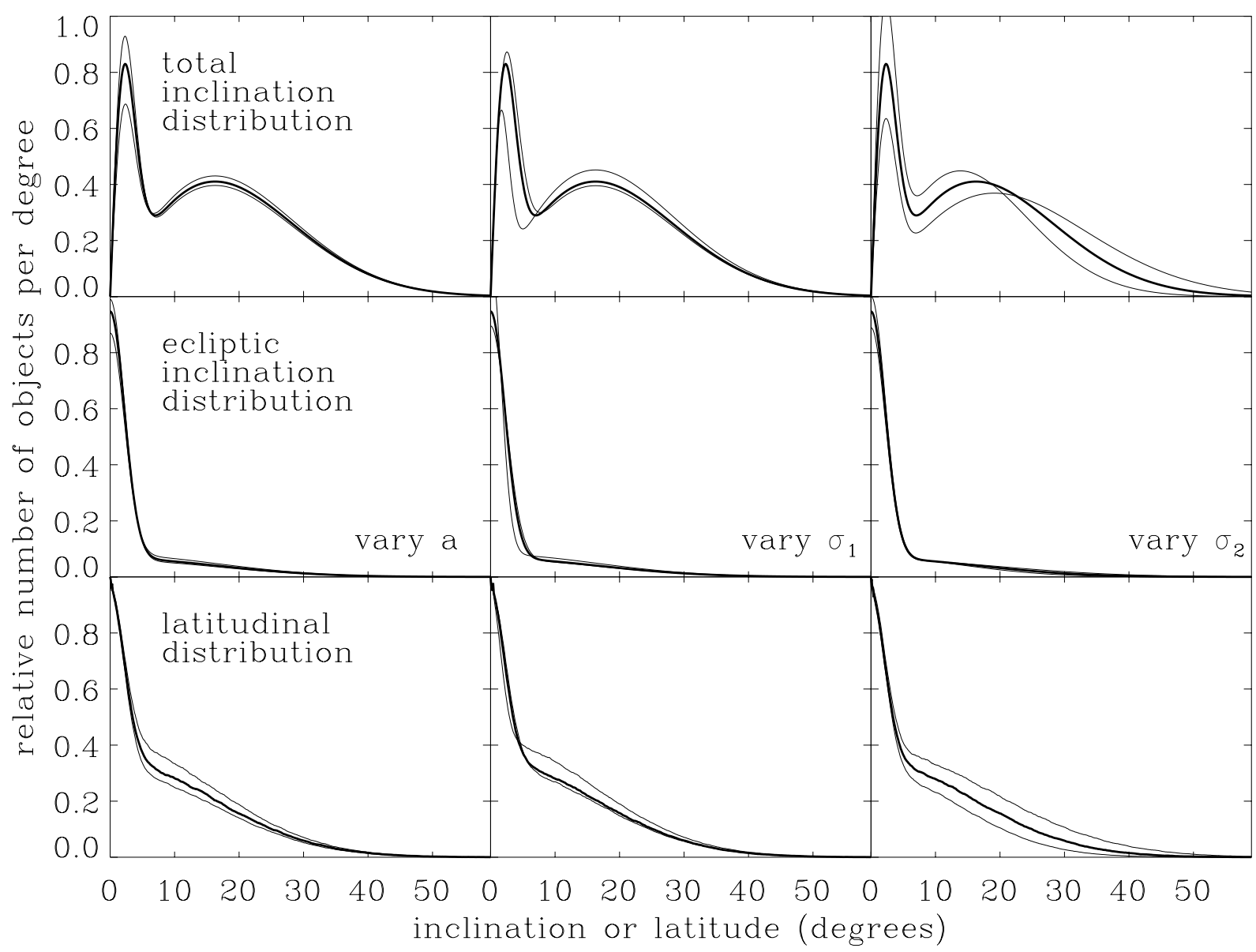

FIG. 12.-Total inclination, ecliptic inclination, and latitudinal distribution of the classical Kuiper belt. For each distribution, we show the $1 \sigma$ variations obtained by holding two of the three parameters constant and allowing the other one to vary. While this method gives an indication of the range of variability allowed, the true range can best be seen from the contour plots of confidence levels.

each known KBO. We thus implicitly take into account all of the biases, inefficiencies, and search regions of the surveys that found the real KBOs. We simulate inclination distributions as single Gaussians multiplied by $\sin i$ with widths

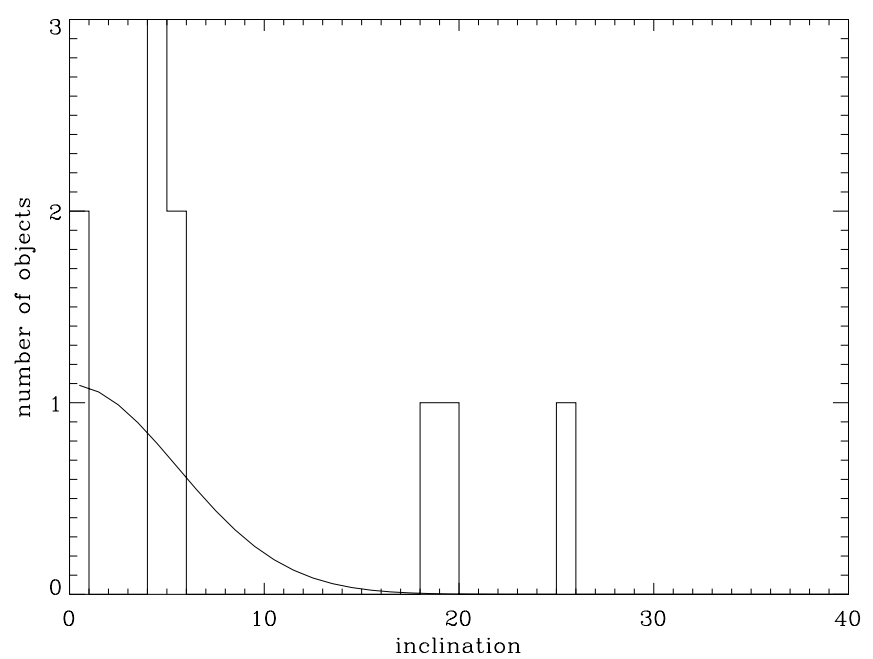

FIG. 13.- Inclination distribution of the nine scattered KBOs found at latitudes lower than 0.5 . The solid line shows the best single-Gaussian fit to the data. With the small number of objects in the distribution, the true form of $f_{e}(i)$ is difficult to discern. between $5^{\circ}$ and $20^{\circ}$. In all cases, we find that the recovered inclination distribution is within $1 \sigma$ of the true inclination distribution.

To explore the effects of even higher eccentricities, we simulate the Plutino population using all the same parameters above, except we allow the eccentricity to range from 0.1 to 0.6 . In this case, we still recover the correct inclination distribution within $1 \sigma$ for all Gaussian widths between $5^{\circ}$ and $20^{\circ}$, although the fits are not as good. If we take the extreme case of allowing eccentricities to vary from 0 to 1 , the best-fit Gaussian is always within $25 \%$ of the true value, although at times the fits are sufficiently bad that we would reject them at the $1 \sigma$ (although never $2 \sigma$ ) level. We thus conclude that our method is robust for the Plutinos, even for eccentricities significantly more extreme than those known to be present.

For the scattered objects, we construct a Monte Carlo population of $10^{6}$ objects with perihelion chosen uniformly between 30 and $40 \mathrm{AU}$, eccentricity chosen between 0 and 1 (Fig. 6), and all other parameters chosen randomly. For Gaussian widths between $10^{\circ}$ and $30^{\circ}$, we again find that the best-fit Gaussian is always within $25 \%$ of the true value, and as before, at times the fits are sufficiently bad that we would reject them at the $1 \sigma$ (although never $2 \sigma$ ) level. We conclude that while our general method works least well for the case of the scattered KBOs and their extreme eccentricities, even for these objects, the method is sufficiently robust 


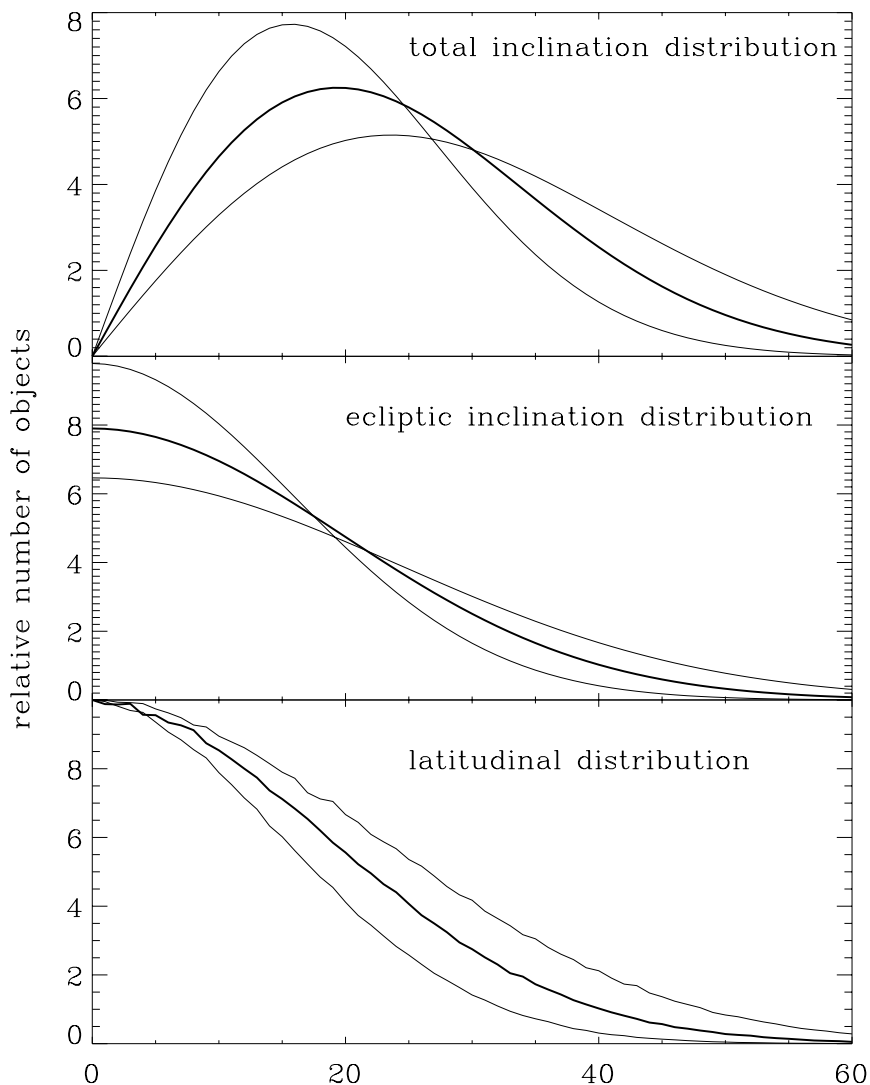

FIG. 14.-Total inclination, ecliptic inclination, and latitudinal distribution of the scattered Kuiper belt. The solid lines show the best-fit distributions, while the thinner lines now show the $2 \sigma$ variations, since all of the distributions can be rejected at the $1 \sigma$ level. Our simulations suggest that these distributions could nonetheless be accurate representations of the true distributions.

that we can have confidence that the final answer is giving physically meaningful results. The relatively poor fit to the real scattered KBO data (Fig. 15) could simply be a consequence of the large range of scattered $\mathrm{KBO}$ eccentricities.

An additional limitation of the results comes not from the method itself, but from the potential errors in the orbital parameters of the known KBOs. Because we use data from all detected KBOs, including those with poorly determined small-arc orbits, we are including some objects of each dynamical class in the wrong class for the analysis. These misclassified objects are those most likely to have significantly different inclinations than those initially reported (Fig. 2). Note in particular that all multiopposition objects that were initially reported to have inclinations above $33^{\circ}$

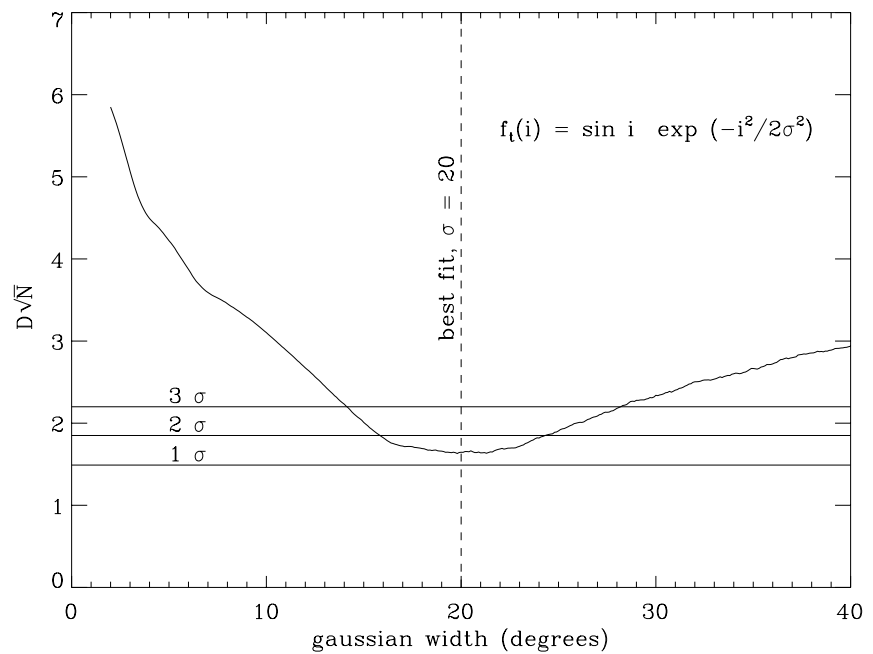

FIG. 15. $-D \sqrt{N}$ statistic as a function of $\sigma$ for $\sin i$ multiplied by a single-Gaussian fit to $f_{t}(i)$ for the scattered KBOs. The best-fit value of $\sigma=20^{\circ}$ can be formally rejected at the $93 \%$ confidence level, but we find from full Monte Carlo simulations of scattered KBO populations that this type of poor fit is expected because of the large eccentricities of these objects. We thus conclude that this single Gaussian is an adequate fit to the data.

are now recognized to have lower inclinations. It is possible that most or all the single-opposition objects with these high inclinations actually are misclassified objects with lower inclinations. It is also possible that single-opposition objects that were searched for and not recovered have inclinations different from those assumed. To determine the likely magnitude of the effects of these problems, we have redone the entire analysis using only objects with multiopposition observations. While the distribution of such objects has its own intrinsic set of biases, we find that the final results are not changed (although the statistics are poorer) by using this smaller sample. Specifically, we find for the Plutinos that $\sigma=10^{\circ} 3_{-3.5}^{+4.8}\left(\mathrm{FWHM}=23^{\circ} \pm 9^{\circ}\right.$, and the effective area is $9600 \pm 3500 \mathrm{deg}^{2}$ ) and for the classical KBOs $a=0.95 \pm 0.03, \sigma_{1}=2.2 \pm 0.8$, and $\sigma_{2}=20^{\circ}$ $\pm 7^{\circ}\left(\mathrm{FWHM}=6.2 \pm 3^{\circ}\right.$, and the effective area is 6200 $\pm 3000 \mathrm{deg}^{2}$ ). The accidental misclassification of objects and errors in initial inclinations appears to have little effect on our final answers.

\section{DISCUSSION}

Figure 16 and Table 1 summarize the results for all classes of KBOs. We discuss the specific implications below.

Total number of KBOs.-Previous estimates of the total number of KBOs (Jewitt, Luu, \& Chen 1996; Gladman et

TABLE 1

SUMMARY OF BEST-Fit KBO Distributions

\begin{tabular}{ccccccccc}
\hline \hline Population & Number & \multicolumn{1}{c}{$a^{\mathrm{a}}$} & $\sigma_{1}$ & $\sigma_{2}$ & $f_{1}{ }^{\mathrm{b}}$ & $f_{2}{ }^{\mathrm{c}}$ & $A_{e}{ }^{\mathrm{d}}$ & FWHM \\
\hline All objects ...... & 379 & $0.83 \pm 0.03$ & $2.6_{-.2}^{+.8}$ & $15 \pm 1$ & 0.26 & 0.74 & $8100_{-1100}^{+1500}$ & $12.5 \pm 3.5$ \\
Plutinos ....... & 70 & 1.00 & $10.2_{-. .8}^{+2.5}$ & $\ldots$ & 1.00 & $\ldots$ & $9300 \pm 1800$ & $23 \pm 5$ \\
Classical ....... & 251 & $0.93 \pm 0.02$ & $2.2_{-.6}^{+.2}$ & $17 \pm 3$ & 0.19 & 0.81 & $6100 \pm 2100$ & $6.8_{-3.6}^{+2.0}$ \\
Scattered ....... & 50 & 1.00 & $20 \pm 4$ & $\ldots$ & 1.00 & $\ldots$ & $17000 \pm 3000$ & $44 \pm 10$ \\
\hline
\end{tabular}

\footnotetext{
${ }^{a}$ Best fit to a total inclination distribution of the form $f_{t}(i)=\sin i\left[a \exp \left(-i^{2} / 2 \sigma_{1}^{2}\right)+(1-a) \exp \left(-i^{2} / 2 \sigma_{2}^{2}\right)\right]$.

${ }^{b}$ Fraction of objects in the narrow Gaussian distribution.

c Fraction of objects in the wide Gaussian distribution.

${ }^{d}$ Effective area.
} 


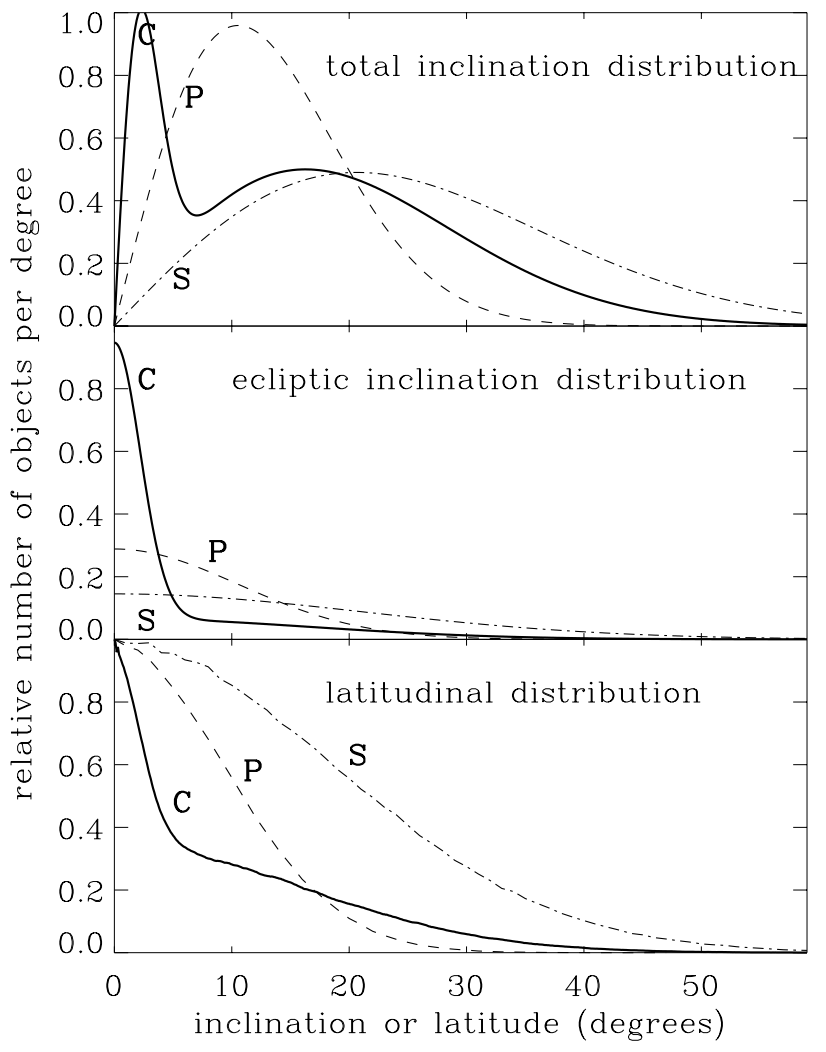

FIG. 16. - Comparison of the best-fit total inclination, ecliptic inclination, and latitudinal distribution of the Plutinos (P), classical KBOs (C) and scattered KBOs (S). The total and ecliptic inclination distributions are normalized to have the same area, while the latitudinal distribution is normalized to unity at $0^{\circ}$ latitude.

al. 1998; Chiang \& Brown 1999) have had to rely on order of magnitude estimates of the effective area of the total Kuiper belt. Following Jewitt et al. (1996), a total area of $10^{4} \mathrm{deg}^{2}$ is usually assumed. This value is almost within the $1 \sigma$ error limits of our estimate of $8100_{-1100}^{+1500} \mathrm{deg}^{2}$. We conclude that the total population of KBOs calculated does not need to be significantly revised.

Plutinos.-Malhotra (1995) found that the inclinations and eccentricities of KBOs can be raised by the resonant capture of an outwardly migrating Neptune. The inclinations in Malhotra's simulations do not reach values as high as those observed, however. Most of the objects in these simulations stay in relatively low-inclination orbits, while a small fraction of them $(\sim 10 \%)$ are pumped to values as high as $20^{\circ}$. In contrast, we find that approximately half of the objects have inclinations above $10^{\circ}$, and $15 \%$ have inclinations higher than $20^{\circ}$. Similar simulations by Ida et al. (2000) verify the lack of high inclinations caused by Neptune's migration. It remains possible that migration and capture is the main mechanism responsible for the high inclinations of the Plutinos - e.g., Malhotra finds a correlation between higher inclinations and slower migration of Neptune-but more work on additional inclinationpumping mechanisms is clearly required.

Classical KBOs.-The classical KBOs are often thought of as being essentially undisturbed since formation in a loweccentricity, low-inclination disk. The extremely narrow component of the inclination distribution likely corresponds to this initial population. However, a significant fraction of the objects that we classified as classical KBOs have been greatly disturbed. The similarity between the width of this component of the classical Kuiper belt and the width of the inclination distribution of the scattered Kuiper belt leads us to investigate the possibility that the classical Kuiper belt is interspersed with scattered KBOs that happen to have small semimajor axes. As one test of this possibility, we exclude all KBOs with perihelia between 30 and $40 \mathrm{AU}$ and redo the complete analysis. We still find that a second wide Gaussian is required to fit the inclinations. Thus the high inclinations do not appear to be associated with unrecognized scattered KBOs. Indeed, a plot of inclination versus eccentricity for the classical KBOs (Fig. 17) shows that the inclinations of the known KBOs are distributed over a much larger range than the eccentricities, even though the observational biases tend to select for the detection of low-inclination, high-eccentricity objects. Most excitation mechanisms would tend to increase inclinations and eccentricities by approximately equal amounts, so we would initially expect to see many more high-eccentricity objects based on the high inclinations. However, objects in such orbits are dynamically unstable (Duncan, Levison, \& Budd 1995), so it is possible that many were excited into such orbits and have now escaped the solar system.

Several mechanisms for the general excitation of the classical Kuiper belt objects have been explored, including planetary migration, excitation by large planetesimals, and stellar encounters. Models of planetary migration show that some classical KBOs have their inclinations increased even when they are not resonantly captured by Neptune, but in all cases, the increases are limited to $5^{\circ}-10^{\circ}$ (Malhotra 1995; Hahn \& Malhotra 1999; Ida et al. 2000) and cannot reproduce the observed inclinations. Large planetesimals moving through the Kuiper belt can also excite eccentricities and inclinations of the KBOs. Morbidelli \& Valsecchi (1997) and Petit, Morbidelli, \& Valsecchi (1999) have modeled several cases of the excitations of such planetesimals. While none of the modeled cases comes close to creating a population of high-inclination classical KBOs, inclusion of suffi-

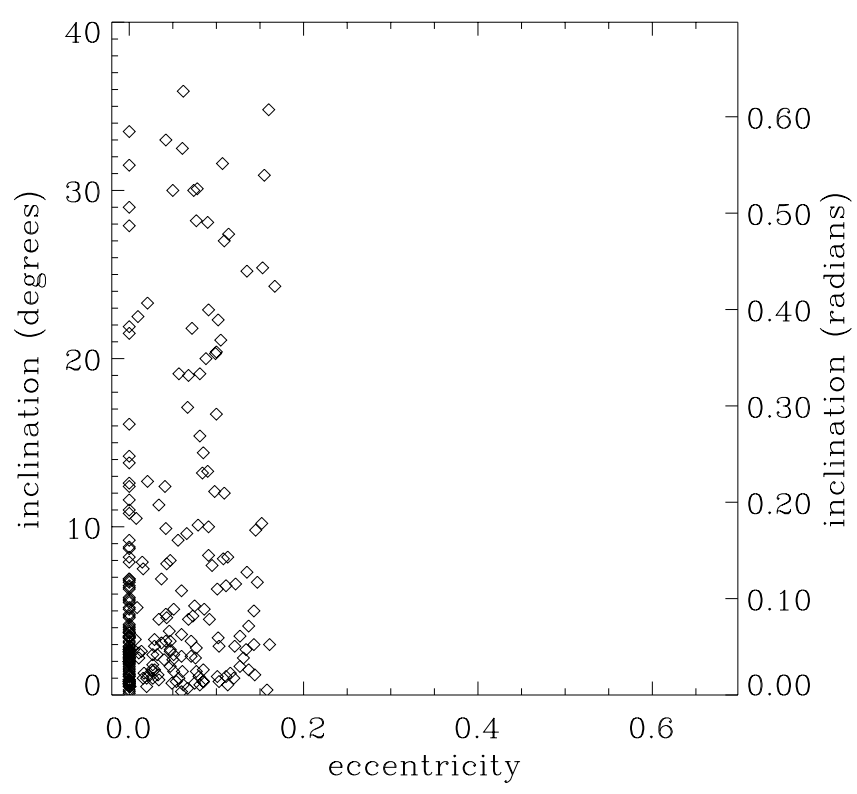

FIG. 17.-Eccentricity vs. inclination for the classical KBOs. In general, random perturbations affect inclination (in radians) and eccentricity equally. Objects with inclinations as high as $30^{\circ}$ correspond to those with eccentricities as high as 0.5 , of which none exist. 
ciently massive scattered objects could greatly increase the inclinations of many objects. Such massive objects would also have the effect of removing objects from the 3:2 resonance, however (Morbidelli \& Valsecchi 1997), so such massive objects appear inconsistent with the observed Kuiper belt. The final mechanism considered for exciting inclinations and eccentricities is a stellar encounter early in the history of the solar system. Such an encounter would more strongly affect distant objects, so the Plutinos could be unperturbed (Ida et al. 2000), although the existence of any objects in the 1:2 resonance becomes difficult. Nonetheless, the simulations of such encounters show an inclination distribution reminiscent of what we have found for the classical objects; a high-inclination population is created, while maintaining a sizable population of low inclinations. In the particular simulations shown, the number of highinclination objects is still too small, but this results could come from tuning the models to produce the current eccentricity distribution without taking into account the fact that high-eccentricity objects could have been created but would have been lost over the age of the solar system. We therefore deem this scenario a promising possiblity for explaining the existence of the high-inclination classical Kuiper belt objects.

Recently, Tegler \& Romanishin (2000) have shown evidence that also suggests that the classical Kuiper belt might be divided into at least two populations, one with red colors and low inclinations and one with more neutral colors and higher inclinations. In addition, Levison \& Stern (2001) have suggested that the classical Kuiper belt is divided into a low-inclination fainter population and a high-inclination brighter population. Both of these effects could be related to the two-component inclination distribution that we find here.

Scattered KBOs.- The broad inclination and latitudinal distributions of the scattered KBOs are consistent with formation by scattering by Neptune. The simulation of Duncan \& Levison (1997) predicts upper limits to the inclinations of the scattered Kuiper belt objects of $\sim 35^{\circ}$. Our best-fit distribution has approximately $20 \%$ of the objects beyond this upper limit; indeed, one known Kuiper belt object appears to have an inclination of $40^{\circ}$. Again, some mechanism appears to be operating, which increases the inclinations beyond that expected, although in this region the statistics and results are sufficiently poor that this conclusion is not firm.

\section{CONCLUSION}

We have developed a method that can be used to determine the inclination distribution of Kuiper belt objects using all objects that have been found from all surveys, and we have applied this method to find the inclination distributions of the Plutinos and the classical and scattered Kuiper belt objects. We find that the effective area of the Kuiper belt has been estimated correctly in previous studies, but that the inclination distributions of the Kuiper belt contain significantly more high-inclination objects than have been found in any dynamical simulation. This general method will be able to be applied easily to continue to refine the inclination distributions of the Kuiper belt as larger numbers of objects are found.
Chiang, E. I., \& Brown, M. E. 1999, AJ, 118, 1411

Duncan, M. J., \& Levison, H. F. 1997, Science, 276, 1670

Duncan, M. J., Levison, H. F., \& Budd, S. M. 1995, AJ, 110, 3073

Gladman, B., Kavelaars, J. J., Nicholson, P. D., Loredo, T. J., \& Burns, J. A. 1998, AJ, 116, 2042

Hahn, J. M., \& Malhotra, R. 1999, AJ, 117, 3041

Ida, S., Bryden, G., Lin, D. N. C., \& Tanaka, H. 2000, ApJ, 534, 428

Jewitt, D., Luu, J., \& Chen, J. 1996, AJ, 112, 1225

Jewitt, D. C., \& Luu, J. X. 2000, in Protostars and Planets IV, ed. V. Mannings, A. P. Boss, \& S. S. Russell (Tuscon: Univ. Arizona Press), 1201

\section{REFERENCES}

Levison, H., \& Stern, S. A. 2001, AJ, 121, 1730

Malhotra, R. 1995, AJ, 110, 420

Morbidelli, A., \& Valsecchi, G. B. 1997, Icarus, 128, 464

Petit, J.-M., Morbidelli, A., \& Valsecchi, G. B. 1999, Icarus, 141, 367

Press, W. H., Teukolsky, S. A., Vetterling, W. T., \& Flannery, B. P. 1992, Numerical Recipes in Fortran (2d ed.; Cambridge: Cambridge Univ. Press)

Tegler, S. C., \& Romanishin, W. 2000, Nature, 407, 979

Trujillo, C. A., Jewitt, D. C., \& Luu, J. X. 2000, ApJ, 529, L103 